\title{
APPROXIMATION AND GENERALIZED GROWTH OF SOLUTIONS TO A CLASS OF ELLIPTIC PARTIAL DIFFERENTIAL EQUATIONS
}

\author{
Susheel Kumar, Girja S. Srivastava
}

\begin{abstract}
In the present paper, we study the approximation and growth of solutions to a class of elliptic partial differential equations. The characterizations of generalized order and generalized type of solutions to a class of elliptic partial differential equations have been obtained in terms of approximation errors.
\end{abstract}

Keywords: Helmholtz type equation, regular solution, analytic function, approximation errors, generalized order, generalized type.

\section{Introduction}

Following McCoy [4], we first give some definitions. A Helmholtz type equation is given by

$$
£[H]:=\left[\partial_{r r}+r^{-1} \partial_{r}+r^{-2} \partial_{\theta \theta}+F\left(r^{2}\right)\right] H(r, \theta)=0 .
$$

Here $(r, \theta)$ are polar coordinates and $F$ is an entire function. Let $H(r, \theta)=$ $H\left(r, e^{i \theta}\right)$ be a regular solution of (1.1) in some sufficiently small star-shaped neighborhood $\Omega$ about origin. Let $R$ be the radius of convergence of this regular solution. Following Bergman [1], we have

$$
H(r, \theta)=\mathbb{B}[f(z)]=\int_{-1}^{+1} E\left(r^{2}, t\right) f(\sigma) d \mu(t),
$$

where $z=r e^{i \theta} \in \Omega, \sigma=z\left(1-t^{2}\right) / 2, d \mu(t)=\left(1-t^{2}\right)^{-1 / 2} d t$, and the associated function $f$ is analytic for $2 z \in \Omega$. The Taylor series expansion of the kernel $E\left(r^{2}, t\right)$ is given as

$$
E\left(r^{2}, t\right)=1+\sum_{n=1}^{\infty} t^{2 n} Q^{(2 n)}\left(r^{2}\right) .
$$

2010 Mathematics Subject Classification: primary: 30B10; secondary: 30D20, 32K05 
For a fixed $r \geqslant 0$, the kernel $E\left(r^{2}, t\right)$ is analytic for $t \in[-1,+1]$ and for every fixed $t \in[-1,+1]$, it is entire for $r \geqslant 0$. The Taylor coefficients $Q^{(2 n)}\left(r^{2}\right)$ are entire function solutions of the system

$$
\begin{gathered}
\frac{\partial\left(Q^{(2)}\left(r^{2}\right)\right)}{\partial r^{2}}+2 F\left(r^{2}\right)=0, \quad Q^{(0)}\left(r^{2}\right)=1, \\
(2 n+1) \frac{\partial\left(Q^{(2 n+2)}\left(r^{2}\right)\right)}{\partial r^{2}}+2 \frac{\partial\left(r^{2} Q^{(2 n)}\left(r^{2}\right)\right)}{\partial r^{2}} \\
+F\left(r^{2}\right) Q^{(2 n)}\left(r^{2}\right)-n \frac{\partial\left(Q^{(2 n)}\left(r^{2}\right)\right)}{\partial r^{2}}=0, \\
\left.Q^{(2 n+2)}\left(r^{2}\right)\right|_{r=0}=0, \quad n=1,2,3 \ldots
\end{gathered}
$$

McCoy [4] defined the basic set of particular solutions

$$
\Phi_{n}\left(r, e^{i \theta}\right)=\left[r^{n} G_{n}\left(r^{2}\right) / R^{n} G_{n}\left(R^{2}\right)\right] e^{i n \theta}
$$

normalized by the conditions

$$
\Phi_{n}\left(r, e^{i \theta}\right)=e^{i n \theta}, \quad n=0,1,2,3 \ldots
$$

Here

$$
G_{n}\left(r^{2}\right)=\int_{-1}^{+1} E\left(r^{2}, t\right)\left(1-t^{2}\right)^{n} d \mu(t) .
$$

This set is complete relative to compact convergence on a disk $D_{R}=\{z:|z|<R\}$. Let $\operatorname{Im}\left(D_{R}\right)$ be the space of regular solutions of (1.1) on $D_{R}$. Then $H \in \operatorname{Im}\left(D_{R}\right)$ has the expansion in a uniformly convergent series

$$
H\left(r, e^{i \theta}\right)=\sum_{n=0}^{\infty} a_{n} \Phi_{n}\left(r, e^{i \theta}\right),
$$

where $\left\{a_{n}\right\}$ is a sequence of real numbers. If $A\left(D_{R}\right)$ is the space of analytic functions on $D_{R}$, then $f \in A\left(D_{R}\right)$ has the Taylor series expansion

$$
f(z)=\sum_{n=0}^{\infty} a_{n} z^{n}, \quad z \in D_{R} .
$$

McCoy [4] associated $H$ with the analytic function $f$ by defining an integral operator as given below:

$$
H\left(r, e^{i \theta}\right)=T_{\varepsilon}[f(z)]=\frac{1}{2 \pi i} \int_{|\zeta|=1-\varepsilon} K_{R}(\zeta) f(z / \zeta) d \zeta / \zeta, \quad z=r e^{i \theta} / R
$$


where $\varepsilon>0$ is arbitrarily small. The kernel for this integral operator defined over the basis $\left\{\Phi_{n}\right\}$ is given by

$$
K_{R}(\zeta)=\sum_{n=0}^{\infty} \zeta^{n}\left[G_{n}\left(r^{2}\right) / G_{n}\left(R^{2}\right)\right] .
$$

For $\varepsilon>0$, there exists an integer $N(\varepsilon)$ such that for all $n \geqslant N(\varepsilon)$, we have

$$
(1-\varepsilon) \leqslant\left|G_{n}\left(r^{2}\right) / G_{n}\left(R^{2}\right)\right| \leqslant(1+\varepsilon) .
$$

Thus we can say that the kernel of this operator has uniformly convergent expansion. The above integral operator maps the function $f \in A\left(D_{R(1-\varepsilon)}\right)$ onto regular solution $H \in \operatorname{Im}\left(D_{R(1-\varepsilon)}\right)$ and the disk of regularity of $H$ coincides with the disk of analyticity of $f$. The maximum modulus of $H$ on $D_{r}$ is given by

$$
M(r, H)=\max \left\{\left|H\left(s, e^{i \theta}\right)\right|: s \leqslant r\right\} .
$$

Let $H$ be regular on the closure $\Delta^{*}$ of the unit disk $\Delta=\{z:|z|<1\}$ and define the norm of $H$ as

$$
\|H\|=\left\{\begin{array}{l}
\|H\|_{p}=\left[\iint_{\Delta^{*}}|H|^{p} r d r d \theta\right]^{1 / p}, \quad 1 \leqslant p<\infty \\
\|H\|_{\infty}=\lim _{r \rightarrow 1^{-}} M(r, H) .
\end{array}\right.
$$

The spaces of polynomial solutions of fixed degree $n=0,1,2, \ldots$ are given by

$$
\Pi_{n}=\left\{P: P\left(r, e^{i \theta}\right)=\sum_{k=0}^{n} c_{k} \Phi_{k}\left(r, e^{i \theta}\right), c_{k} \in \mathbb{R}\right\} .
$$

We define the approximation errors $E_{n}(H)$ (see [4]) by

$$
E_{n}(H)=\inf _{P}\left\{\|H-P\|: P \in \Pi_{n}\right\}, \quad n=0,1,2 \ldots
$$

The definition of order and type for regular solution $H$ are the same as those for the associated analytic function $f$ (see [4], pp. 209). Hence the order $\rho$ of regular solution $H$ on $D_{R}$ is given by

$$
\rho=\lim _{r \rightarrow R^{-}} \sup \frac{\ln ^{+} \ln ^{+} M(r, H)}{\ln [R /(R-r)]},
$$

where

$$
\ln ^{+} x= \begin{cases}\ln x, & x>1 \\ 0, & 0<x \leqslant 1\end{cases}
$$

Further, for $0<\rho<\infty$ the type $\sigma$ of regular solution $H$ on $D_{R}$ is given by

$$
\sigma=\lim _{r \rightarrow R^{-}} \sup \frac{\ln ^{+} M(r, H)}{[R /(R-r)]^{\rho}} .
$$


McCoy [4] obtained the characterizations of order and type of function $H$ in terms of approximation errors. Later, in [5], using the concept of index, McCoy studied the growth of entire solutions of the Helmoltz equation. Using the concept of $(p, q)$ growth, Kumar [3] studied the relation between the growth and Chebyshev approximation of entire function solutions of Helmoltz equation. Srivastava and Kumar [7] obtained the characterizations of generalized growth of function $H$ in terms of approximation errors and Taylor series coefficients It is clear from the above that the definition of $\sigma$ is not valid if the order $\rho=\infty$. For such cases, following Janik [2] and Seremeta [6] we define the generalized order and generalized type of function $H$. Hence, let $L^{0}$ denote the class of functions $h$ satisfying the following conditions:

(i) $h$ is defined on $[a, \infty)$ and is positive, strictly increasing, differentiable and $h(x)$ tends to $\infty$ as $x \rightarrow \infty$,

(ii) $\lim _{x \rightarrow \infty} \frac{h\{(1+1 / \psi(x)) x\}}{h(x)}=1$, for every function $\psi$ such that $\psi(x) \rightarrow \infty$ as $x$ tends to $\infty$.

(iii) let $\Lambda$ denote the class of functions $h$ satisfying condition (i) and

$$
\lim _{x \rightarrow \infty} \frac{h(c x)}{h(x)}=1, \quad c>0
$$

i.e., $h$ is slowly increasing.

For $\alpha \in \Lambda$ and $\beta \in L^{0}$ we define the generalized order of $H$ as

$$
\rho(\alpha, \beta, H)=\lim _{r \rightarrow R^{-}} \sup \frac{\alpha\left[\ln ^{+} M(r, H)\right]}{\beta[R /(R-r)]} .
$$

Further, for $\alpha, \beta, \gamma \in \Lambda$ and $0<\rho<\infty$, we define the generalized type of $H$ as

$$
\sigma(\alpha, \beta, \gamma, H)=\lim _{r \rightarrow R^{-}} \sup \frac{\alpha\left[\ln ^{+} M(r, H)\right]}{\beta\left\{[\gamma\{R /(R-r)\}]^{\rho}\right\}} .
$$

If $\rho(\alpha, \beta, H)$ defined as above is zero then the analytic function is of generalized order zero and $\sigma(\alpha, \beta, \gamma, H)$ is no longer defined. For such functions we give the modified definition of generalized order. Hence for $\alpha(x) \in \Lambda$, we define the generalized order $\rho(\alpha, H),(0 \leqslant \rho(\alpha, H)<\infty)$ of $H$ on $D_{R}$ as

$$
\rho(\alpha, H)=\lim _{r \rightarrow R^{-}} \sup \frac{\alpha\left[\ln ^{+} M(r, H)\right]}{\alpha[\ln \{R /(R-r)\}]} .
$$

Also for $\beta(x) \in L^{0}$ and $1<\rho(\alpha, H)<\infty$, we define the generalized type $\sigma(\beta, \rho, H)$ of $H$ on $D_{R}$ as

$$
\sigma(\beta, \rho, H)=\lim _{r \rightarrow R^{-}} \sup \frac{\beta\left[\ln ^{+} M(r, H)\right]}{(\beta[\ln \{R /(R-r)\}])^{\rho}} .
$$

In the present paper we have obtained the characterizations of generalized order and type defined by (1.2) and (1.3). We have also obtained the characterizations of generalized slow growth of function $H$ in terms of approximation errors. 


\section{Generalized $(\alpha, \beta)$-growth}

We now prove

Theorem 1. Let $H$ be a regular solution of (1.1) having the series expansion $H\left(r, e^{i \theta}\right)=\sum_{n=0}^{\infty} a_{n} \Phi_{n}\left(r, e^{i \theta}\right)$. For $\alpha \in \Lambda, \beta \in L^{0}$ and positive numbers $x$ and $\mu_{1}$, set $U\left(x, \mu_{1}\right)=\beta^{-1}\left\{\mu_{1} \alpha(x)\right\}$. Assume that $\alpha\left(x / U\left(x, \mu_{1}\right)\right) \cong[1+o(x)] \alpha(x)$ as $x \rightarrow \infty$. Then $H$ is the restriction of a solution $H_{1}$ whose disk of regularity is $D_{R}(R>1)$ and having generalized order $\rho(0<\rho<\infty)$ if and only if

$$
\rho=\rho(\alpha, \beta, H)=\lim _{n \rightarrow \infty} \sup \frac{\alpha(n)}{\beta\left\{n / \ln ^{+}\left(E_{n}(H) R^{n}\right)\right\}} .
$$

Proof. Write

$$
\eta_{1}=\lim _{n \rightarrow \infty} \sup \frac{\alpha(n)}{\beta\left\{n / \ln ^{+}\left(E_{n}(H) R^{n}\right)\right\}} .
$$

Now first we prove that $\eta_{1} \leqslant \rho$. From (1.2), for $\mu_{1}>\rho$ and $r$ sufficiently close to $R$, we have

$$
\log ^{+} M\left(r, H_{1}\right) \leqslant \alpha^{-1}\left[\mu_{1} \beta\{R /(R-r)\}\right] .
$$

Let $\varepsilon>0$ be arbitrary such that $v=\left(R^{-1}+\varepsilon\right)<1$. Following McCoy ([4], pp.208), we have

$$
E_{k}(H) \leqslant \frac{\pi K(\varepsilon) v^{k}}{1-v} ; \quad k=n, n+1, \ldots
$$

where $K(\varepsilon)$ is a finite positive number. Let us put $r=v^{-1}$. Then $1<r<R$. For sufficiently small $\varepsilon, r$ is close to $R$ and $\pi K(\varepsilon) \leqslant M(r, H)$. Hence we have

$$
E_{k}(H) \leqslant \frac{M(r, H)}{(r-1) r^{k-1}} \leqslant \frac{M\left(r, H_{1}\right)}{(r-1) r^{k-1}}, \quad 1<r<R, k \geqslant n .
$$

Hence for every $r$ sufficiently close to $R$ and large $n$, we get

$$
\ln ^{+}\left(E_{n}(H) R^{n}\right) \leqslant O(1)-n \ln (r / R)+\alpha^{-1}\left[\mu_{1} \beta\{R /(R-r)\}\right] .
$$

Putting

$$
r=r_{n}=R\left[1-1 / U\left(n / U\left(n, \mu_{1}^{-1}\right), \mu_{1}^{-1}\right)\right]
$$

we get

$$
\ln ^{+}\left(E_{n}(H) R^{n}\right) \leqslant O(1)-n \ln \left[1-1 / U\left(n / U\left(n, \mu_{1}^{-1}\right), \mu_{1}^{-1}\right)\right]+n / U\left(n, \mu_{1}^{-1}\right) .
$$

Now using the properties of logarithm and assumptions of the theorem for $\alpha(x)$ and $\beta(x)$, we get for sufficiently large values of $n$, 


$$
\ln ^{+}\left(E_{n}(H) R^{n}\right) \leqslant C_{1} \frac{n}{\beta^{-1}\left\{\mu_{1}^{-1} \alpha(n)\right\}},
$$

where $C_{1}$ is a positive constant. Hence by using the properties of $\beta$, we get

$$
\frac{\alpha(n)}{\beta\left\{n / \ln ^{+}\left(E_{n}(H) R^{n}\right)\right\}} \leqslant \mu_{1}
$$

Now proceeding to limits as $n \rightarrow \infty$, we get $\eta_{1} \leqslant \mu_{1}$. Since $\mu_{1}>\rho$ is arbitrary, therefore we get $\eta_{1} \leqslant \rho$.

Now we will prove that $\rho \leqslant \eta_{1}$. Let us assume that $0 \leqslant \eta_{1}<\infty$ otherwise for $\eta_{1}=\infty$, the inequality obviously holds. Therefore for a given $\varepsilon>0$ there exists a positive integer $n_{0}$ such that for all $n>n_{0}$, we have

$$
0 \leqslant \frac{\alpha(n)}{\beta\left\{n / \ln ^{+}\left(E_{n}(H) R^{n}\right)\right\}} \leqslant \eta_{1}+\varepsilon=\eta_{1}^{*}
$$

or

$$
E_{n}(H) r^{n} \leqslant r^{n} R^{-n} \exp \left[n / \beta^{-1}\left\{\left(\eta_{1}^{*}\right)^{-1} \alpha(n)\right\}\right] .
$$

Now from the property of maximum modulus, we have

$$
M(r, H) \leqslant \sum_{n=0}^{\infty} E_{n}(H) r^{n}
$$

or

$$
M(r, H) \leqslant \sum_{n=0}^{n_{0}} E_{n}(H) r^{n}+\sum_{n=n_{0}+1}^{\infty} r^{n} R^{-n} \exp \left[n / \beta^{-1}\left\{\left(\eta_{1}^{*}\right)^{-1} \alpha(n)\right\}\right]
$$

or

$$
M(r, H) \leqslant A_{1} r^{n_{0}}+\sum_{n=n_{0}+1}^{\infty} r^{n} R^{-n} \exp \left[n / \beta^{-1}\left\{\left(\eta_{1}^{*}\right)^{-1} \alpha(n)\right\}\right],
$$

where $A_{1}$ is a positive real constant. We take

$$
N(r)=\left[\alpha^{-1}\left(\eta_{1}^{*} \beta\left\{[\ln \{R /(N+1) r\}]^{-1}\right\}\right)\right],
$$

where $[x]$ denotes the integer part of $x \geqslant 0$. Since $\alpha \in \Lambda$ and $\beta \in L^{0}$, the integer $N(r)$ is well defined. Now if $r$ is sufficiently large, then from (2.4) we have

$$
\begin{aligned}
M(r, H) \leqslant & A_{1} r^{n_{0}}+r^{N(r)} \sum_{n_{0}+1 \leqslant n \leqslant N(r)} R^{-n} \exp \left[n / \beta^{-1}\left\{\left(\eta_{1}^{*}\right)^{-1} \alpha(n)\right\}\right] \\
& +\sum_{n>N(r)} r^{n} R^{-n} \exp \left[n / \beta^{-1}\left\{\left(\eta_{1}^{*}\right)^{-1} \alpha(n)\right\}\right]
\end{aligned}
$$


or

$$
\begin{aligned}
M(r, H) \leqslant & A_{1} r^{n_{0}}+r^{N(r)} \sum_{n=1}^{\infty} R^{-n} \exp \left[n / \beta^{-1}\left\{\left(\eta_{1}^{*}\right)^{-1} \alpha(n)\right\}\right] \\
& +\sum_{n>N(r)} r^{n} R^{-n} \exp \left[n / \beta^{-1}\left\{\left(\eta_{1}^{*}\right)^{-1} \alpha(n)\right\}\right]
\end{aligned}
$$

Now we have

$$
\lim _{n \rightarrow \infty} \sup \left(R^{-n} \exp \left[n / \beta^{-1}\left\{\left(\eta_{1}^{*}\right)^{-1} \alpha(n)\right\}\right]\right)^{1 / n}=\frac{1}{R}<1 .
$$

Hence the first series on right hand side of (2.6) converges to a positive real constant $A_{2}$. So from (2.6) we get

$$
M(r, H) \leqslant A_{1} r^{n_{0}}+A_{2} r^{N(r)}+\sum_{n>N(r)} r^{n} R^{-n} \exp \left[n / \beta^{-1}\left\{\left(\eta_{1}^{*}\right)^{-1} \alpha(n)\right\}\right]
$$

or

$$
M(r, H) \leqslant A_{1} r^{n_{0}}+A_{2} r^{N(r)}+\sum_{n>N(r)} r^{n} R^{-n} \exp [n \ln \{R /(N+1) r\}]
$$

or

$$
M(r, H) \leqslant A_{1} r^{n_{0}}+A_{2} r^{N(r)}+\sum_{n>N(r)}\left(\frac{1}{N+1}\right)^{n}
$$

or

$$
M(r, H) \leqslant A_{1} r^{n_{0}}+A_{2} r^{N(r)}+\sum_{n=1}^{\infty}\left(\frac{1}{N+1}\right)^{n} .
$$

It can be easily seen that the series in (2.7) converges to a positive real constant $A_{3}$. Therefore from (2.7), we get

$$
M(r, H) \leqslant A_{2} r^{N(r)}[1+o(1)]
$$

or

$$
\ln ^{+} M(r, H) \leqslant[1+o(1)]\left[\alpha^{-1}\left(\overline{\eta_{1}} \beta\left\{[\ln \{R /(N+1) r\}]^{-1}\right\}\right)\right] \ln r
$$

or

$$
\ln ^{+} M(r, H) \leqslant[1+o(1)] \alpha^{-1}\left[\left\{\eta_{1}^{*}+\delta_{1}\right\} \beta\left\{[\ln \{R /(N+1) r\}]^{-1}\right\}\right],
$$

where $\delta_{1}>0$ is suitably small. Hence

$$
\alpha\left[\ln ^{+} M(r, H)\right] \leqslant\left\{\eta_{1}^{*}+\delta_{1}\right\} \beta\left\{[1+o(1)]^{-1}[\ln (R / r)]^{-1}\right\} .
$$

Thus for $r$ sufficiently close to $R$, we get

$$
\frac{\alpha\left[\ln ^{+} M(r, H)\right]}{\beta\left\{[1+o(1)]^{-1}[R /(R-r)]\right\}} \leqslant \eta_{1}^{*}+\delta_{1} .
$$


Proceeding to limits as $r \rightarrow R$ and using the property of $\beta$, we get

$$
\lim _{r \rightarrow R^{-}} \sup \frac{\alpha\left[\ln ^{+} M(r, H)\right]}{\beta\{R /(R-r)\}} \leqslant \eta_{1}^{*}+\delta_{1} .
$$

Since $\varepsilon$ and $\delta_{1}$ are arbitrarily small, therefore finally we get $\rho \leqslant \eta_{1}$. Combining this with the earlier inequality obtained, we get $\rho=\eta_{1}$.

Now from (2.2), for every $\lambda_{1}>\eta_{1}$ and for sufficiently large $n$, we have

$$
\frac{\alpha(n)}{\beta\left\{n / \ln ^{+}\left(E_{n}(H) R^{n}\right)\right\}} \leqslant \lambda_{1}
$$

or

$$
E_{n}(H) R^{n} \leqslant \exp \left[n / \beta^{-1}\left\{\lambda_{1}^{-1} \alpha(n)\right\}\right] .
$$

Hence proceeding to limits as $n \rightarrow \infty$, we get

$$
\lim _{n \rightarrow \infty} \sup \left(E_{n}(H) R^{n}\right)^{1 / n} \leqslant 1 .
$$

Since $\eta_{1}>0$, the sequence $\left(E_{n}(H) R^{n}\right)_{n \in \mathbb{N}}$ is unbounded, whence

$$
\lim _{n \rightarrow \infty} \sup \left(E_{n}(H) R^{n}\right)^{1 / n} \geqslant 1 .
$$

Hence finally we get

$$
\lim _{n \rightarrow \infty} \sup \left(E_{n}(H) R^{n}\right)^{1 / n}=1 .
$$

Thus following McCoy ([4], Theorem 1) we claim that the regular solution $H$ can be continuously extended to a regular solution whose disk of regularity is $D_{R}(R>1)$.

Let us put

$$
H_{1}\left(r, e^{i \theta}\right)=\sum_{n=0}^{\infty} E_{n}(H) \Phi_{n}\left(r, e^{i \theta}\right) .
$$

Now we show that $H_{1}$ is the required continuation of $H$ and $\rho\left(\alpha, \beta, H_{1}\right)=\eta_{1}$. For every $\lambda_{1}>\eta_{1}$ and for sufficiently large $n$, we have

$$
E_{n}(H) R^{n} \leqslant \exp \left[n / \beta^{-1}\left\{\lambda_{1}^{-1} \alpha(n)\right\}\right] .
$$

Now as in the proof of this theorem (see (2.4) to (2.7) above), we claim that

$$
\rho\left(\alpha, \beta, H_{1}\right) \leqslant \lambda_{1} .
$$

Since $\lambda_{1}>\eta_{1}$ is arbitrary, so we get

$$
\rho\left(\alpha, \beta, H_{1}\right) \leqslant \eta_{1} .
$$

Also following the proof of first part given above, we get

$$
\eta_{1} \leqslant \rho\left(\alpha, \beta, H_{1}\right)
$$

Hence finally we get $\rho\left(\alpha, \beta, H_{1}\right)=\eta_{1}$. This completes the proof of Theorem 1 . 
Next we prove

Theorem 2. Let $H$ be a regular solution of (1.1) and have the series expansion $H\left(r, e^{i \theta}\right)=\sum_{n=0}^{\infty} a_{n} \Phi_{n}\left(r, e^{i \theta}\right)$. For positive $x, \mu_{2}$ and $\rho$, we set

$$
V\left(x, \mu_{2}, \rho\right)=\gamma^{-1}\left\{\left[\beta^{-1}\left(\mu_{2} \alpha(x)\right)\right]^{1 / \rho}\right\} .
$$

Assume that for $\alpha(x), \beta(x), \gamma(x) \in \Lambda$,

$$
V\left(\frac{n(\rho+1)}{\rho V\left(n / \rho, 1 / \mu_{2}, \rho+1\right)}, \frac{1}{\mu_{2}}, \rho\right) \cong[1+o(n)] V\left(n / \rho, 1 / \mu_{2}, \rho+1\right) \quad \text { as } x \rightarrow \infty .
$$

Then $H$ is the restriction of a solution $H_{1}$ whose disk of regularity is $D_{R}(R>1)$ and having generalized type $\sigma(0<\sigma<\infty)$ if and only if

$$
\sigma=\sigma\left(\alpha, \beta, \gamma, H_{1}\right)=\lim _{n \rightarrow \infty} \sup \frac{\alpha(n / \rho)}{\beta\left\{\left[\gamma\left\{(\rho+1)\left[\rho \ln ^{+}\left(E_{n}(H) R^{n}\right)^{1 / n}\right]^{-1}\right\}\right]^{(\rho+1)}\right\}}
$$

Proof. Write

$$
\eta_{2}=\lim _{n \rightarrow \infty} \sup \frac{\alpha(n / \rho)}{\beta\left\{\left[\gamma\left\{(\rho+1)\left[\rho \ln ^{+}\left(E_{n}(H) R^{n}\right)^{1 / n}\right]^{-1}\right\}\right]^{(\rho+1)}\right\}}
$$

Now first we prove that $\eta_{2} \leqslant \sigma$. From (1.3), for $\mu_{2}>\sigma$ and $r$ sufficiently close to $R$, we have

$$
\ln ^{+} M\left(r, H_{1}\right) \leqslant \alpha^{-1}\left[\mu_{2} \beta\left\{[\gamma\{R /(R-r)\}]^{\rho}\right\}\right] .
$$

Thus as in the proof of Theorem 1 , here we have

$$
\ln ^{+}\left(E_{n}(H) R^{n}\right) \leqslant O(1)-n \ln (r / R)+\alpha^{-1}\left[\mu_{2} \beta\left\{[\gamma\{R /(R-r)\}]^{\rho}\right\}\right] .
$$

Putting

$$
r=r_{n}=R\left[1-\left\{V\left(\frac{n(\rho+1)}{\rho V\left(n / \rho, 1 / \mu_{2}, \rho+1\right)}, \frac{1}{\mu_{2}}, \rho\right)\right\}^{-1}\right]
$$

we get

$$
\begin{aligned}
\ln ^{+}\left(E_{n}(H) R^{n}\right) \leqslant & O(1)-n \ln \left[1-\left\{V\left(\frac{n(\rho+1)}{\rho V\left(n / \rho, 1 / \mu_{2}, \rho+1\right)}, \frac{1}{\mu_{2}}, \rho\right)\right\}^{-1}\right] \\
& +n \frac{\rho+1}{\rho}\left[\gamma^{-1}\left\{\left[\beta^{-1}\left\{\mu_{2}^{-1} \alpha(n / \rho)\right\}\right]^{1 /(\rho+1)}\right\}\right]^{-1} .
\end{aligned}
$$


Now using the properties of logarithm and assumptions of theorem, we get for sufficiently large values of $n$

$$
\ln ^{+}\left(E_{n}(H) R^{n}\right) \leqslant C_{2} n \frac{\rho+1}{\rho}\left[\gamma^{-1}\left\{\left[\beta^{-1}\left\{\mu_{2}^{-1} \alpha(n / \rho)\right\}\right]^{1 /(\rho+1)}\right\}\right]^{-1}
$$

where $C_{2}$ is a positive constant. Hence by using the properties of $\alpha, \beta$ and $\gamma$, we get

$$
\frac{\alpha(n / \rho)}{\beta\left\{\left[\gamma\left\{(\rho+1)\left[\rho \ln ^{+}\left(E_{n}(H) R^{n}\right)^{1 / n}\right]^{-1}\right\}\right]^{(\rho+1)}\right\}} \leqslant \mu_{2} .
$$

Now proceeding to limits as $n \rightarrow \infty$ we get $\eta_{2} \leqslant \mu_{2}$. Since $\mu_{2}>\sigma$ is arbitrary, therefore finally we get $\eta_{2} \leqslant \sigma$. Now we will prove that $\sigma \leqslant \eta_{2}$. If $\eta_{2}=\infty$, then there is nothing to prove. So let us assume that $0 \leqslant \eta_{2}<\infty$. Therefore for a given $\varepsilon>0$ there exists $n_{0} \in N$ such that for all $n>n_{0}$, we have

$$
0 \leqslant \frac{\alpha(n / \rho)}{\beta\left\{\left[\gamma\left\{(\rho+1)\left[\rho \log ^{+}\left(E_{n}(H) R^{n}\right)^{1 / n}\right]^{-1}\right\}\right]^{(\rho+1)}\right\}} \leqslant \eta_{2}+\varepsilon=\eta_{2}^{*}
$$

or

$$
E_{n}(H) R^{n} \leqslant \exp \left\{n \frac{\rho+1}{\rho}\left[\gamma^{-1}\left\{\left[\beta^{-1}\left\{\left(\eta_{2}^{*}\right)^{-1} \alpha(n / \rho)\right\}\right]^{1 /(\rho+1)}\right\}\right]^{-1}\right\}
$$

or

$$
E_{n}(H) r^{n} \leqslant r^{n} R^{-n} \exp \left\{n \frac{\rho+1}{\rho}\left[\gamma^{-1}\left\{\left[\beta^{-1}\left\{\left(\eta_{2}^{*}\right)^{-1} \alpha(n / \rho)\right\}\right]^{1 /(\rho+1)}\right\}\right]^{-1}\right\} .
$$

Now from the property of maximum modulus, we have

$$
\begin{aligned}
M(r, H) & \leqslant \sum_{n=0}^{\infty} E_{n}(H) r^{n} \\
\leqslant & \sum_{n=0}^{n_{0}} E_{n}(H) r^{n} \\
& +\sum_{n=n_{0}+1}^{\infty} r^{n} R^{-n} \exp \left\{n \frac{\rho+1}{\rho}\left[\gamma^{-1}\left\{\left[\beta^{-1}\left\{\left(\eta_{2}^{*}\right)^{-1} \alpha(n / \rho)\right\}\right]^{1 /(\rho+1)}\right\}\right]^{-1}\right\}
\end{aligned}
$$

or

$$
\begin{aligned}
M(r, H) \leqslant & B_{1} r^{n_{0}}+\sum_{n=n_{0}+1}^{\infty} r^{n} R^{-n} \\
& \times \exp \left\{n \frac{\rho+1}{\rho}\left[\gamma^{-1}\left\{\left[\beta^{-1}\left\{\left(\eta_{2}^{*}\right)^{-1} \alpha(n / \rho)\right\}\right]^{1 /(\rho+1)}\right\}\right]^{-1}\right\},
\end{aligned}
$$


where $B_{1}$ is a positive real constant. We take

$$
N(r)=\left[\rho \alpha^{-1}\left\{\eta_{2}^{*} \beta\left(\left[\gamma\left\{(\rho+1)[\rho \ln \{R /(N+1) r\}]^{-1}\right\}\right]^{(\rho+1)}\right)\right\}\right],
$$

where $[x]$ denotes the integer part of $x \geqslant 0$. Since $\alpha(x), \beta(x), \gamma(x) \in \Lambda$, the integer $N(r)$ is well defined. Now if $r$ is sufficiently close to $R$, then from (2.10) we have

$$
\begin{aligned}
& M(r, H) \leqslant B_{1} r^{n_{0}} \\
& +r^{N(r)} \sum_{n_{0}+1 \leqslant n \leqslant N(r)} R^{-n} \exp \left\{n \frac{\rho+1}{\rho}\left[\gamma^{-1}\left\{\left[\beta^{-1}\left\{\left(\eta_{2}^{*}\right)^{-1} \alpha(n / \rho)\right\}\right]^{1 /(\rho+1)}\right\}\right]^{-1}\right\} \\
& +\sum_{n>N(r)} r^{n} R^{-n} \exp \left\{n \frac{\rho+1}{\rho}\left[\gamma^{-1}\left\{\left[\beta^{-1}\left\{\left(\eta_{2}^{*}\right)^{-1} \alpha(n / \rho)\right\}\right]^{1 /(\rho+1)}\right\}\right]^{-1}\right\}
\end{aligned}
$$

or

$$
\begin{aligned}
& M(r, H) \leqslant B_{1} r^{n_{0}} \\
& +r^{N(r)} \sum_{n=1}^{\infty} R^{-n} \exp \left\{n \frac{\rho+1}{\rho}\left[\gamma^{-1}\left\{\left[\beta^{-1}\left\{\left(\eta_{2}^{*}\right)^{-1} \alpha(n / \rho)\right\}\right]^{1 /(\rho+1)}\right\}\right]^{-1}\right\} \\
& \quad+\sum_{n>N(r)} r^{n} R^{-n} \exp \left\{n \frac{\rho+1}{\rho}\left[\gamma^{-1}\left\{\left[\beta^{-1}\left\{\left(\eta_{2}^{*}\right)^{-1} \alpha(n / \rho)\right\}\right]^{1 /(\rho+1)}\right\}\right]^{-1}\right\} .
\end{aligned}
$$

Now we have

$$
\begin{aligned}
\lim _{n \rightarrow \infty} \sup \left(R^{-n} \exp \left\{n \frac{\rho+1}{\rho}\left[\gamma^{-1}\left\{\left[\beta^{-1}\left\{\left(\eta_{2}^{*}\right)^{-1} \alpha(n / \rho)\right\}\right]^{1 /(\rho+1)}\right\}\right]^{-1}\right\}\right)^{1 / n} & =\frac{1}{R}<1
\end{aligned}
$$

Hence the first series in (2.11) converges to a positive real constant $B_{2}$. Hence from (2.11), we get

$$
\begin{aligned}
M(r, H) \leqslant & B_{1} r^{n_{0}}+B_{2} r^{N(r)} \\
& +\sum_{n>N(r)} r^{n} R^{-n} \exp \left\{n \frac{\rho+1}{\rho}\left[\gamma^{-1}\left\{\left[\beta^{-1}\left\{\left(\eta_{2}^{*}\right)^{-1} \alpha(n / \rho)\right\}\right]^{1 /(\rho+1)}\right\}\right]^{-1}\right\}
\end{aligned}
$$

or

$$
M(r, H) \leqslant B_{1} r^{n_{0}}+B_{2} r^{N(r)}+\sum_{n>N(r)} r^{n} R^{-n} \exp [n \ln \{R /(N+1) r\}]
$$

or

$$
M(r, H) \leqslant B_{1} r^{n_{0}}+B_{2} r^{N(r)}+\sum_{n>N(r)}\left(\frac{1}{N+1}\right)^{n}
$$


or

$$
M(r, H) \leqslant B_{1} r^{n_{0}}+B_{2} r^{N(r)}+\sum_{n=1}^{\infty}\left(\frac{1}{N+1}\right)^{n} .
$$

It can be easily seen that the series in (2.12) converges to a positive real constant $B_{3}$. Therefore from (2.12), we get

$$
M(r, H) \leqslant B_{1} r^{n_{0}}+B_{2} r^{N(r)}+B_{3} \leqslant B_{2} r^{N(r)}[1+o(1)]
$$

or

$$
\begin{aligned}
\ln ^{+} M(r, H) \leqslant & {[1+o(1)] } \\
& \times\left[\rho \alpha^{-1}\left\{\eta_{2}^{*} \beta\left(\left[\gamma\left\{(\rho+1)[\rho \ln \{R /(N+1) r\}]^{-1}\right\}\right]^{(\rho+1)}\right)\right\}\right] \ln r
\end{aligned}
$$

or

$$
\begin{aligned}
\ln ^{+} M(r, H) \leqslant & {[1+o(1)] } \\
& \times\left[\alpha^{-1}\left\{\left(\eta_{2}^{*}+\delta_{2}\right) \beta\left(\left[\gamma\left\{(\rho+1)[\rho \ln \{R /(N+1) r\}]^{-1}\right\}\right]^{(\rho+1)}\right)\right\}\right],
\end{aligned}
$$

where $\delta_{2}>0$ is suitably small. Hence

$$
\alpha\left[\ln ^{+} M(r, H)\right] \leqslant\left(\eta_{2}^{*}+\delta_{2}\right) \beta\left(\left[\gamma\left\{(\rho+1)[\rho \ln \{R /(N+1) r\}]^{-1}\right\}\right]^{(\rho+1)}\right) .
$$

When $r$ is sufficiently close to $R$, then by using properties of $\beta$ and $\gamma$, we get

$$
\frac{\alpha\left[\ln ^{+} M(r, H)\right]}{\beta\left\{[\gamma\{R /(R-r)\}]^{\rho}\right\}} \leqslant \eta_{2}^{*}+\delta_{2} .
$$

Since $\varepsilon$ and $\delta_{2}$ are arbitrarily small, proceeding to limits as $r \rightarrow R^{-}$, we get

$$
\sigma \leqslant \eta_{2}
$$

Now as in Theorem 1 we can similarly prove that the regular solution $H$ can be continuously extended to a regular solution whose disk of regularity is $D_{R}(R>1)$. Let us put

$$
H_{1}\left(r, e^{i \theta}\right)=\sum_{n=0}^{\infty} E_{n}(H) \Phi_{n}\left(r, e^{i \theta}\right) .
$$

Now we claim that $H_{1}$ is the required continuation of $H$ and $\sigma\left(\alpha, \beta, \gamma, H_{1}\right)=\eta_{2}$. From (2.8), for every $\lambda_{2}>\eta_{2}$ and for sufficiently large $n$, we have

$$
E_{n}(H) R^{n} \leqslant \exp \left\{n \frac{\rho+1}{\rho}\left[\gamma^{-1}\left\{\left[\beta^{-1}\left\{\left(\lambda_{2}\right)^{-1} \alpha(n / \rho)\right\}\right]^{1 /(\rho+1)}\right\}\right]^{-1}\right\}
$$

Now as in the proof of this theorem (see (2.9) to (2.13)), we claim that

$$
\sigma\left(\alpha, \beta, \gamma, H_{1}\right) \leqslant \lambda_{2} .
$$


Since $\lambda_{2}>\eta_{2}$ is arbitrary, so finally we get

$$
\sigma\left(\alpha, \beta, \gamma, H_{1}\right) \leqslant \eta_{2}
$$

Also following the proof of first part given above, we get

$$
\eta_{2} \leqslant \sigma\left(\alpha, \beta, \gamma, H_{1}\right)
$$

Hence finally we get $\sigma\left(\alpha, \beta, \gamma, H_{1}\right)=\eta_{2}$. This completes the proof of Theorem 2 .

\section{Functions of generalized slow growth}

In this section we give the characterizations of generalized order and type for functions of slow growth. We have

Theorem 3. Let $H$ be a regular solution of (1.1) and have the series expansion $H\left(r, e^{i \theta}\right)=\sum_{n=0}^{\infty} a_{n} \Phi_{n}\left(r, e^{i \theta}\right)$. Then for $\alpha(x) \in \Lambda, H$ is a restriction of a solution $H_{1}$ whose disk of regularity is $D_{R}(R>1)$ and having generalized order $\rho\left(\alpha, H_{1}\right)$ if and only if

$$
\rho\left(\alpha, H_{1}\right)=\lim _{n \rightarrow \infty} \sup \frac{\alpha(n)}{\alpha\left[\log ^{+}\left\{n / \ln ^{+}\left(E_{n}(H) R^{n}\right)\right\}\right]} .
$$

Proof. First we assume that $H$ has an extension $H_{1}$ whose disk of regularity is $D_{R}(R>1)$ and is of generalized order $\rho\left(\alpha, H_{1}\right)$. We write $\rho\left(\alpha, H_{1}\right)=\rho$ and

$$
\zeta_{1}=\lim _{n \rightarrow \infty} \sup \frac{\alpha(n)}{\alpha\left[\log ^{+}\left\{n / \ln ^{+}\left(E_{n}(H) R^{n}\right)\right\}\right]} .
$$

First we prove that $\zeta_{1} \leqslant \rho$. As shown above, from (2.3) we have

$$
E_{k}(H) \leqslant \frac{M(r, H)}{(r-1) r^{k-1}}, \quad 1<r<R, k \geqslant n
$$

Also using (1.4), for arbitrarily small $\varepsilon>0$ and $r>r_{0}(\varepsilon)$, we have

$$
M(r, H) \leqslant \exp \left(\alpha^{-1}\left\{\rho^{*} \alpha[\ln \{R /(R-r)\}]\right\}\right),
$$

where $\rho^{*}=\rho+\varepsilon$. From (3.2) and (3.3), we get

$$
\ln ^{+}\left(E_{n}(H) R^{n}\right) \leqslant-\ln ^{+}(r-1)-n \ln ^{+}(r / R)+\alpha^{-1}\left\{\rho^{*} \alpha[\ln \{R /(R-r)\}]\right\} .
$$

Write $F\left(x, c_{1}\right)=\alpha^{-1}\left\{c_{1} \alpha(x)\right\}$, where $x$ and $c_{1}$ are positive real numbers. Now putting $r=r_{n}$, where

$$
r_{n}=R\left(1-\left[\exp \left\{F\left(n / \exp \left\{F\left(n,\left(\rho^{*}\right)^{-1}\right)\right\},\left(\rho^{*}\right)^{-1}\right)\right\}\right]^{-1}\right)
$$


we get

$$
\begin{aligned}
\ln ^{+}\left(E_{n}(H) R^{n}\right) \leqslant & -\ln ^{+}\left(r_{n}-1\right) \\
& -n \ln ^{+}\left(1-\left[\exp \left\{F\left(n / \exp \left\{F\left(n,\left(\rho^{*}\right)^{-1}\right)\right\},\left(\rho^{*}\right)^{-1}\right)\right\}\right]^{-1}\right) \\
& +n / \exp \left\{F\left(n,\left(\rho^{*}\right)^{-1}\right)\right\} .
\end{aligned}
$$

Now using the properties of logarithm, we get for sufficiently large value of $n$

$$
\ln ^{+}\left(E_{n}(H) R^{n}\right) \leqslant\{1+o(1)\}\left[n / \exp \left\{F\left(n,\left(\rho^{*}\right)^{-1}\right)\right\}\right] .
$$

From the above inequality, we get

$$
\alpha^{-1}\left\{\left(\rho^{*}\right)^{-1} \alpha(n)\right\} \leqslant\{1+o(1)\} \ln ^{+}\left\{n / \ln ^{+}\left(E_{n}(H) R^{n}\right)\right\}
$$

or

$$
\alpha(n) \leqslant \rho^{*} \alpha\left[\{1+o(1)\} \ln ^{+}\left\{n / \ln ^{+}\left(E_{n}(H) R^{n}\right)\right\}\right]
$$

or

$$
\frac{\alpha(n)}{\alpha\left[\ln ^{+}\left\{n / \ln ^{+}\left(E_{n}(H) R^{n}\right)\right\}\right]} \leqslant \rho^{*} \frac{\alpha\left[\{1+o(1)\} \ln ^{+}\left\{n / \ln ^{+}\left(E_{n}(H) R^{n}\right)\right\}\right]}{\alpha\left[\ln ^{+}\left\{n / \ln ^{+}\left(E_{n}(H) R^{n}\right)\right\}\right]} .
$$

Proceeding to limits as $n \rightarrow \infty$ and using the properties of $\alpha(x)$, we get $\zeta_{1} \leqslant \rho^{*}$. Since $\varepsilon>0$ is arbitrarily small, we finally get $\zeta_{1} \leqslant \rho$.

Now we will prove that $\rho \leqslant \zeta_{1}$. If $\zeta_{1}=\infty$, then there is nothing to prove. So let us assume that $0 \leqslant \zeta_{1}<\infty$. Therefore for a given $\varepsilon>0$ there exists $n_{0} \in N$ such that for all $n>n_{0}$, we have

$$
0 \leqslant \frac{\alpha(n)}{\alpha\left[\log ^{+}\left\{n / \log ^{+}\left(E_{n}(H) R^{n}\right)\right\}\right]} \leqslant \zeta_{1}+\varepsilon=\zeta_{1}^{*}
$$

or

$$
E_{n}(H) R^{n} \leqslant \exp \left\{n / \exp \left[\alpha^{-1}\left\{\left(\zeta_{1}^{*}\right)^{-1} \alpha(n)\right\}\right]\right\}
$$

or

$$
E_{n}(H) r^{n} \leqslant r^{n} R^{-n} \exp \left\{n / \exp \left[\alpha^{-1}\left\{\left(\zeta_{1}^{*}\right)^{-1} \alpha(n)\right\}\right]\right\} .
$$

Now from the property of maximum modulus, we have

$$
\begin{aligned}
M\left(r, H_{1}\right) \leqslant & \sum_{n=0}^{\infty} E_{n}(H) r^{n} \leqslant \sum_{n=0}^{n_{0}} E_{n}(H) r^{n} \\
& +\sum_{n=n_{0}+1}^{\infty} r^{n} R^{-n} \exp \left\{n / \exp \left[\alpha^{-1}\left\{\left(\zeta_{1}^{*}\right)^{-1} \alpha(n)\right\}\right]\right\}
\end{aligned}
$$


or

$$
M\left(r, H_{1}\right) \leqslant A_{1} r^{n_{0}}+\sum_{n=n_{0}+1}^{\infty} r^{n} R^{-n} \exp \left\{n / \exp \left[\alpha^{-1}\left\{\left(\zeta_{1}^{*}\right)^{-1} \alpha(n)\right\}\right]\right\},
$$

where $A_{1}$ is a positive real constant. We take

$$
W_{1}(r)=\left[\alpha^{-1}\left\{\zeta_{1}^{*} \alpha\left[\ln \left\{\ln \left[R /\left(\delta_{1}+1\right) r\right]\right\}^{-1}\right]\right\}\right],
$$

where $\delta_{1}>0$ is arbitrarily small and $[x]$ denotes the integer part of $x \geqslant 0$. Since $\alpha(x) \in \Lambda$, the integer $W_{1}(r)$ is well defined. Now if $r$ is sufficiently large, then from $(3.5)$, we have

$$
\begin{aligned}
M\left(r, H_{1}\right) \leqslant & A_{1} r^{n_{0}}+r^{W_{1}(r)} \\
& \times \sum_{n_{0}+1 \leqslant n \leqslant W_{1}(r)} R^{-n} \exp \left\{n / \exp \left[\alpha^{-1}\left\{\left(\zeta_{1}^{*}\right)^{-1} \alpha(n)\right\}\right]\right\} \\
& +\sum_{n>W_{1}(r)} r^{n} R^{-n} \exp \left\{n / \exp \left[\alpha^{-1}\left\{\left(\zeta_{1}^{*}\right)^{-1} \alpha(n)\right\}\right]\right\}
\end{aligned}
$$

or

$$
\begin{aligned}
M\left(r, H_{1}\right) \leqslant & A_{1} r^{n_{0}}+r^{W_{1}(r)} \sum_{n=1}^{\infty} R^{-n} \exp \left\{n / \exp \left[\alpha^{-1}\left\{\left(\zeta_{1}^{*}\right)^{-1} \alpha(n)\right\}\right]\right\} \\
& +\sum_{n>W_{1}(r)} r^{n} R^{-n} \exp \left\{n / \exp \left[\alpha^{-1}\left\{\left(\zeta_{1}^{*}\right)^{-1} \alpha(n)\right\}\right]\right\} .
\end{aligned}
$$

Now we have

$$
\lim _{n \rightarrow \infty} \sup \left(R^{-n} \exp \left\{n / \exp \left[\alpha^{-1}\left\{\left(\zeta_{1}^{*}\right)^{-1} \alpha(n)\right\}\right]\right\}\right)^{1 / n}=\frac{1}{R}<1 .
$$

Hence the first series in (3.6) converges to a positive real constant $A_{2}$. So from (3.6), we get

$$
\begin{aligned}
M\left(r, H_{1}\right) \leqslant & A_{1} r^{n_{0}}+A_{2} r^{W_{1}(r)} \\
& +\sum_{n>W_{1}(r)} r^{n} R^{-n} \exp \left\{n / \exp \left[\alpha^{-1}\left\{\left(\zeta_{1}^{*}\right)^{-1} \alpha(n)\right\}\right]\right\} \\
\leqslant & A_{1} r^{n_{0}}+A_{2} r^{W_{1}(r)}+\sum_{n>W_{1}(r)} r^{n} R^{-n} \exp \left[n \ln \left\{R /\left(\delta_{1}+1\right) r\right\}\right] \\
\leqslant & A_{1} r^{n_{0}}+A_{2} r^{W_{1}(r)}+\sum_{n>W_{1}(r)}\left[1 /\left(\delta_{1}+1\right)\right]^{n}
\end{aligned}
$$

or

$$
M\left(r, H_{1}\right) \leqslant A_{1} r^{n_{0}}+A_{2} r^{W_{1}(r)}+\sum_{n=1}^{\infty}\left[1 /\left(\delta_{1}+1\right)\right]^{n} .
$$


It can be easily seen that the series in (3.7) converges to a positive real constant $A_{3}$. Therefore from (3.7), we get

$$
M\left(r, H_{1}\right) \leqslant A_{1} r^{n_{0}}+A_{2} r^{W_{1}(r)}+A_{3} \leqslant A_{2} r^{W_{1}(r)}[1+o(1)]
$$

or

$$
\begin{aligned}
\ln ^{+} M\left(r, H_{1}\right) & \leqslant[1+o(1)] W_{1}(r) \ln r \\
& \leqslant[1+o(1)]\left[\alpha^{-1}\left\{\zeta_{1}^{*} \alpha\left[\ln \left\{\ln \left[R /\left(\delta_{1}+1\right) r\right]\right\}^{-1}\right]\right\}\right] \ln r \\
& \leqslant O(1)\left[\alpha^{-1}\left\{\zeta_{1}^{*} \alpha\left[\ln \left\{\ln \left[R /\left(\delta_{1}+1\right) r\right]\right\}^{-1}\right]\right\}\right] .
\end{aligned}
$$

Since $\delta_{1}>0$ is arbitrarily small, for $r$ sufficiently close to $R$, we get

$$
\ln ^{+} M\left(r, H_{1}\right) \leqslant O(1)\left[\alpha^{-1}\left\{\zeta_{1}^{*} \alpha[\ln \{R /(R-r)\}]\right\}\right]
$$

or

$$
\alpha\left[\ln ^{+} M\left(r, H_{1}\right)\right] \leqslant \zeta_{1}^{*} \alpha[\ln \{R /(R-r)\}]+O(1)
$$

Thus for $r$ sufficiently close to $R$, we get

$$
\frac{\alpha\left[\ln ^{+} M\left(r, H_{1}\right)\right]}{\alpha[\ln \{R /(R-r)\}]} \leqslant \zeta_{1}^{*}+o(1) .
$$

Proceeding to limits as $r \rightarrow R^{-}$, we get

$$
\rho \leqslant \zeta_{1}^{*}
$$

Since $\varepsilon>0$ is arbitrarily small, therefore finally we get

$$
\rho \leqslant \zeta_{1}
$$

Now from (3.1), for every $\lambda_{1}>\zeta_{1}$ and for sufficiently large value of $n$, we have

$$
\frac{\alpha(n)}{\alpha\left[\log ^{+}\left\{n / \log ^{+}\left(E_{n}(H) R^{n}\right)\right\}\right]} \leqslant \lambda_{1}
$$

or

$$
E_{n}(H) R^{n} \leqslant \exp \left\{n / \exp \left[\alpha^{-1}\left\{\left(\lambda_{1}\right)^{-1} \alpha(n)\right\}\right]\right\} .
$$

Now for sufficiently large value of $n$, we get

$$
\left[E_{n}(H) R^{n}\right]^{1 / n} \leqslant 1
$$

Proceeding to limits as $n \rightarrow \infty$, we get

$$
\lim _{n \rightarrow \infty} \sup \left[E_{n}(H) R^{n}\right]^{1 / n} \leqslant 1 .
$$


Since $\eta_{1}>0$, the sequence $\left(E_{n}(H) R^{n}\right)_{n \in \mathbb{N}}$ is unbounded, whence

$$
\lim _{n \rightarrow \infty} \sup \left[E_{n}(H) R^{n}\right]^{1 / n} \geqslant 1 \text {. }
$$

Hence finally we get

$$
\lim _{n \rightarrow \infty} \sup \left[E_{n}(H) R^{n}\right]^{1 / n}=1 .
$$

Thus following McCoy ([4], Theorem 1) we claim that the regular solution $H$ can be continuously extended to a regular solution whose disk of regularity is $D_{R}(R>1)$. Let us put

$$
H_{1}\left(r, e^{i \theta}\right)=\sum_{n=0}^{\infty} E_{n}(H) \Phi_{n}\left(r, e^{i \theta}\right) .
$$

Now we claim that $H_{1}$ is the required continuation of $H$ and $\rho\left(\alpha, H_{1}\right)=\zeta_{1}$. For every $\lambda_{1}>\zeta_{1}$ and for sufficiently large value of $n$, we have

$$
E_{n}(H) R^{n} \leqslant \exp \left\{n / \exp \left[\alpha^{-1}\left\{\left(\lambda_{1}\right)^{-1} \alpha(n)\right\}\right]\right\}
$$

Now as in the proof of this theorem $[(3.4)$ to $(3.8)]$, we claim that

$$
\rho\left(\alpha, H_{1}\right) \leqslant \lambda_{1}
$$

Since $\lambda_{1}>\zeta_{1}$ is arbitrary, so we get

$$
\rho\left(\alpha, H_{1}\right) \leqslant \zeta_{1}
$$

Also following the proof of first part given above, we get

$$
\zeta_{1} \leqslant \rho\left(\alpha, H_{1}\right)
$$

So finally we get

$$
\rho\left(\alpha, H_{1}\right)=\zeta_{1}
$$

This completes the proof of Theorem 3 .

Next we have

Theorem 4. Let $H$ be a regular solution of (1.1) and have the series expansion $H\left(r, e^{i \theta}\right)=\sum_{n=0}^{\infty} a_{n} \Phi_{n}\left(r, e^{i \theta}\right)$. Then for $1<\rho<\infty$ and $\beta(x) \in L^{0}, H$ is a restriction of a solution $H_{1}$ whose disk of regularity is $D_{R}(R>1)$ and having generalized type $\sigma\left(\beta, \rho, H_{1}\right)$ if and only if

$$
\sigma\left(\beta, \rho, H_{1}\right)=\lim _{n \rightarrow \infty} \sup \frac{\beta(n)}{\left(\beta\left[\log ^{+}\left\{n / \ln ^{+}\left(E_{n}(H) R^{n}\right)\right\}\right]\right)^{\rho}} .
$$

Proof. The proof of the above theorem follows on the lines of proof of Theorem 2 and Theorem 3. Hence we omit the proof. 
Next we have

Theorem 5. Let $H$ be a regular solution of (1.1) and have the series expansion $H\left(r, e^{i \theta}\right)=\sum_{n=0}^{\infty} a_{n} \Phi_{n}\left(r, e^{i \theta}\right)$. Then for $\alpha(x) \in \Lambda$ the generalized order $\rho(\alpha, H)(0 \leqslant \rho(\alpha, H)<\infty)$ of $H$ is given by

$$
\rho(\alpha, H)=\lim _{n \rightarrow \infty} \sup \frac{\alpha(n)}{\alpha\left[\ln ^{+}\left\{n / \ln ^{+}\left(\left|a_{n}\right| R^{n}\right)\right\}\right]} .
$$

Proof. The proof is similar to Theorem 3 above and ([7], Theorem 2.1). Hence the proof is omitted.

Lastly we have

Theorem 6. Let $H$ be a regular solution of (1.1) and have the series expansion $H\left(r, e^{i \theta}\right)=\sum_{n=0}^{\infty} a_{n} \Phi_{n}\left(r, e^{i \theta}\right)$. Then for $1<\rho<\infty$ and $\beta(x) \in L^{0}$ the generalized type $\sigma(\beta, \rho, H)$ of $H$ is given by

$$
\sigma(\beta, \rho, H)=\lim _{n \rightarrow \infty} \sup \frac{\beta(n)}{\left(\beta\left[\ln ^{+}\left\{n / \ln ^{+}\left(\left|a_{n}\right| R^{n}\right)\right\}\right]\right)^{\rho}} .
$$

Proof. The proof is similar to Theorem 2 above and ([7], Theorem 2.2). Hence the proof is omitted.

Acknowledgement. The authors are very much indebted to the referee for his valuable comments which helped in improving the paper.

\section{References}

[1] S. Bergman, Integral operators in the theory of linear partial differential equations, Ergebnisse der Mathematik und ihrer Grenzgebiete, Band 23, SpringerVerlag, New York, 1969.

[2] A. Janik, On approximation of analytic functions and generalized order, Ann. Polon. Math. 55 (1991), 163-167.

[3] D. Kumar, Growth and Chebyshev approximation of entire function solutions of Helmoltz equation in $\mathbb{R}^{2}$, Eur. J. Pure Appl. Math. 3 (2010), no. 6, 10621069.

[4] P.A. McCoy, Optimal approximation and growth of solutions to a class of elliptic partial differential equations, J. Math. Anal. Appl. 154 (1991), 203211.

[5] P.A. McCoy, Solutions of the Helmoltz equation having rapid growth, Complex Var. Elliptic Equ. 18 (1992), no. 1, 91-101.

[6] M.N. Seremeta, On the connection between the growth of a function analytic in a disc and moduli of the coefficients of its Taylor series, Visnik L'viv. Derzh. Univ. Ser. Mekh. Mat. 2 (1965), 101-110. 
[7] G.S. Srivastava and S. Kumar, Generalized growth of solutions to a class of elliptic partial differential equations, Acta Math. Vietnam. 37 (2012), no. 1, $11-21$.

Addresses: Susheel Kumar: Department of Mathematics, Jaypee University of Engineering and Technology, Guna - 473226 (M. P.), India;

Girja S. Srivastava: Department of Mathematics, Jaypee Institute of Information Technology, Noida-201309 (U. P.), India.

E-mail: sus83dma@gmail.com, gs91490@gmail.com

Received: 25 June 2015; revised: 16 November 2015 